\section{Fataler Mangel an regulatorischen T-Zellen}

\author{
Die atopische Reaktionsbereitschaft ist gekennzeichnet durch eine \\ unphysiologische Dominanz von TH2-Zellen. Bei Nicht-Atopikern \\ werden diese allergenspezifischen Effektorzellen durch regula- \\ torische T-Zellen (Tr1) in Schach gehalten. Diese Wechselwirkungen \\ untersuchte ein Schweizer Forscherteam jetzt genauer.
}

S chon länger ist bekannt, dass auch nicht atopische Personen periphere allergenspezifische T-Zellen aufweisen, die dieselben T-Zell-Epitope erkennen wie die allergenspezifischen T-Zellen von allergischen Patienten. In einer Studie wurden diese Zellen direkt aus dem Blut von Nicht-Atopikern sowie von Birken- und Milben-sensibilisierten Allergikern entsprechend ihrem Zytokinprofil isoliert, nachdem sie mit verschiedenen hochreinen Antigenen, z. B. rBet v 1, rDer p 1, aktiviert worden waren. Auf diese Weise erhielt man Interferon- $\gamma$-, IL-4- und IL-10-produzierende allergenspezifische CD4+Helfer-Zellen, die molekularbiologi- sche Charakteristika vom Typus TH1-, TH2- und Tr1-Zelle zeigten.

Bei den Gesunden dominierten einheitlich die allergenspezifischen Tr1-Zellen. Bei den Allergikern war dagegen die Rate von allergenspezifischen IL-4-produzierenden Zellen, also Zellen vom TH2-Typus, erhöht. Die Tr1-Zellen entfalteten in vitro zahlreiche immunsuppressive Wirkungen, so die Produktion der Zytokine IL-10 und TGF- $\beta$ sowie die Expression der Oberflächenmoleküle CTLA-4 (zytotoxisches T-Lymphozyten-Antigen-4) und PD-1 (Programmed Death-1). Neutralisationsexperimente gegen die vier Moleküle ergaben, dass ihre Anwesenheit die Zahl der allergen- spezifischen TH2-Tellen bei Gesunden offensichtlich herabregulieren kann.

Damit könnten die von regulatorischen T-Zellen synthetisierten Moleküle IL-10, TGF- $\beta$, CTLA- 4 und PD-1 eine wichtige Rolle in der Suppression von allergischen Immunreaktionen spielen. Warum diese regulatorischen T-Zellen beim Allergiker nur ungenügend gebildet werden, ist noch nicht klar. Auf jeden Fall wirkt sich das gestörte Gleichgewicht auf die Ausdifferenzierung der Antigen-präsentierenden Zellen aus, welche wiederum die Bildung von Tr1-Zellen beeinflusst.

Fazit: $\mathrm{Ob}$ jemand allergisch reagiert oder nicht, hängt von dem fein ausbalancierten Gleichgewicht zwischen TH2-Zellen und regulatorischen T-Zellen ab. $\quad b k$

Akdis $\mathbf{M}$ et al. Immune responses in healthy and allergic individuals are characterized by a fine balance between allergen-specific $T$ regulatory 1 and T helper 2 cells. J Exp Med 2004; 199: 1567-75

\section{Aluminiumhydroxid dämpft TH2-Antwort}

\author{
Aluminiumhydroxid ist ein übliches Adjuvans in Präparaten zur \\ spezifischen Immuntherapie (SIT). In Tierversuchen ergaben sich \\ Hinweise auf eine TH2-verstärkende Wirkung dieser anorganischen \\ Substanz, was den Zielen der SIT zuwiderläuft. Doch zeigt sich in \\ In-vitro-Versuchen mit humanen Zellen eine andere Immunantwort.
}

B ei ihren Untersuchungen isolierten britische Forscher die peripheren mononukleären Zellen des Blutes von 18 Graspollenallergikern und stimulierten sie mit einem Phleum-pratense-Extrakt in An- oder Abwesenheit von Aluminiumhydroxid.

Unter der Stimulation mit dem Allergenextrakt plus Aluminiumhydroxid fiel die Produktion von IL-5 und IL-13 signifikant geringer aus als bei einer Stimulation mit Phleum pratense alleine. Die Produktion von IFN- $\gamma$ und IL-12 blieb dagegen konstant. Die Zugabe von neutralisierenden Antikörpern gegen IL4 oder IL-12 hatte keinen Einfluss auf die beobachteten Veränderungen bei den TH2-Zytokinen. Der Zusatz von Aluminiumhydroxid steigerte außerdem die Expression von CD86 und HLA auf den Monozyten, während die Expression von CD80 signifikant abnahm.

Die Reduktion von IL-5 und IL-13 bei Allergenstimulation unter Aluminiumhydroxid-Zusatz spricht für eine $\mathrm{Ab}$ nahme der TH2-Zytokinantwort, wobei dieser immunmodulatorische Effekt offensichtlich nicht auf IL-12- oder IL4-abhängigen Mechanismen beruht. Die Induktion von CD86-Oberflächenmolekülen könnte als Aktivierung von Antigen-präsentierenden Zellen inter- pretiert werden, die geänderte Expression von CD80 steht möglicherweise im Zusammenhang mit einer Herabregelung bestimmter Immunantworten. Die auch unter AluminiumhydroxidZugabe konstant bleibende Produktion der TH1-Zytokine IFN- $\gamma$ und IL12 deutet auf ein TH1-neutrales Verhalten des Adjuvans hin.

Fazit: Im In-vitro-Versuch an isolierten Monozyten atopischer Patienten führt die zusätzliche Gabe von Aluminiumhydroxid bei Allergenstimulation zu einer Herabregulation der TH2-Antwort, die TH1-Antwort wird nicht beeinflusst. Damit scheint das Adjuvans nicht nur einen Depoteffekt für die Allergene zu besitzen, sondern auch direkt in den immunologischen Umstimmungsprozess einzugreifen.

$b k$

Wilcock LK et al. Aluminium hydroxide down-regulates $T$ helper 2 responses by allergen-stimulated human peripheral blood mononuclear cells. Clin Exp Allergy 2004; 34: 1373-8 